\title{
The Intersection of Two Ringed Surfaces and Some Related Problems
}

\author{
Hee-Seok Heo and Sung Je Hong
}

Department of Computer Science, POSTECH, Pohang 790-784, Korea

Joon-Kyung Seong and Myung-Soo Kim ${ }^{1}$

School of Computer Science and Engineering, Seoul National University, Seoul 151-742, Korea

and

\author{
Gershon Elber
}

Computer Science Department, Technion, IIT, Haifa 32000, Israel

Received August 26, 2001

We present an efficient and robust algorithm to compute the intersection curve of two ringed surfaces, each being the sweep $\cup_{u} C^{u}$ generated by a moving circle. Given two ringed surfaces $\cup_{u} C_{1}^{u}$ and $\cup_{v} C_{2}^{v}$, we formulate the condition $C_{1}^{u} \cap C_{2}^{v} \neq \emptyset$ (i.e., that the intersection of the two circles $C_{1}^{u}$ and $C_{2}^{v}$ is nonempty) as a bivariate equation $\lambda(u, v)=0$ of relatively low degree. Except for redundant solutions and degenerate cases, there is a rational map from each solution of $\lambda(u, v)=0$ to the intersection point $C_{1}^{u} \cap C_{2}^{v}$. Thus it is trivial to construct the intersection curve once we have computed the zero-set of $\lambda(u, v)=0$. We also analyze exceptional cases and consider how to construct the corresponding intersection curves. A similar approach produces an efficient algorithm for the intersection of a ringed surface and a ruled surface, which can play an important role in accelerating the ray-tracing of ringed surfaces. Surfaces of linear extrusion and surfaces of revolution reduce their respective intersection algorithms to simpler forms than those for ringed surfaces and ruled surfaces. In particular, the bivariate equation $\lambda(u, v)=0$ is reduced to a decomposable form, $f(u)=g(v)$ or $\|\mathbf{f}(u)-\mathbf{g}(v)\|=|r(u)|$, which can be solved more efficiently than the general case. (c) 2001 Elsevier Science (USA)

\footnotetext{
${ }^{1}$ Corresponding author. E-mail: mskim@cse.snu.ac.kr.
} 


\section{INTRODUCTION}

A ringed surface is a sweep surface generated by a circle moving under translation, rotation, and scaling; thus it can be decomposed into a one-parameter family of circles [10]. Surfaces of revolution, Dupin cyclides, and canal surfaces all belong to the class of ringed surfaces $[15,19]$. Additionally, early investigations of generalized cylinders were focused on the sweeping of circles along a trajectory space curve. In short, we find the ringed surface in various branches of three-dimensional shape modeling and processing. Out of many related results, we mention van Wijk's ray-tracing algorithm for canal surfaces [22] and Nishita and Johan's scan-line algorithm for rendering ringed surfaces [17]; the examples in these two papers include the representation of various interesting three-dimensional shapes by canal and ringed surfaces.

The intersection of two freeform surfaces is another problem of general importance in geometric and solid modeling. For example, Boolean operations on boundary models rely on accurate computation of the intersection curves between the surfaces of the two original solids. Many algorithms have been suggested for computing the intersection of two surfaces. However, it remains a challenging task to develop an algorithm that can compute the intersection curve of two arbitrary freeform surfaces accurately, robustly, efficiently, and without user intervention [9]. Because of the difficulty of dealing with general freeform surfaces, many previous approaches to the intersection problem have been focused on surfaces of common special types such as the classic CSG primitives (planes, cylinders, cones, and tori) $[11,16,21]$.

In this paper, we consider the intersection of two ringed surfaces. The problem is more difficult than the case of natural quadrics or the usual CSG primitives; in fact, cylinders, cones, and tori may be considered the simplest types of ringed surface. On the other hand, ringed surfaces are simpler than general freeform surfaces, and so it is reasonable to look for a simpler solution to the intersection problem in this case.

Dupin cyclides have both implicit and parametric representations. Martin et al. [15] computed the intersection of cyclides with planes, quadrics, and other cyclides by substituting the parametric equation of one surface into the implicit equation of the other. Johnstone [10] presented an algorithm for intersecting a ringed surface with a cyclide, using circle decomposition and a transformation called the inversion map. Johnstone reformulated the intersection of a ringed surface and a cyclide as the intersection of a ruled surface and a one-parameter family of cyclides. The parametric equation for each line of the ruled surface is then substituted into the implicit equation of the corresponding cyclide in the family, producing an algebraic equation in two variables. However, Johnstone leaves open the problem of intersecting two ringed surfaces. In this paper, we attack this problem using an approach similar to the one taken in our previous work on the intersection of two ruled surfaces [8].

Given two ringed surfaces $\cup_{u} C_{1}^{u}$ and $\cup_{v} C_{2}^{v}$, each represented as a one-parameter family of circles, we formulate the condition $C_{1}^{u} \cap C_{2}^{v} \neq \varnothing$ using a bivariate equation $\lambda(u, v)=0$ of relatively low degree. There is a rational map from the zero-set of $\lambda(u, v)=0$ to the intersection curve $\left(\cup_{u} C_{1}^{u}\right) \cap\left(\cup_{v} C_{2}^{v}\right)$, except for some redundant or degenerate cases.

We consider each circle to be the intersection of a sphere and a plane (i.e., $C_{1}^{u}=O_{1}^{u} \cap P_{1}^{u}$ and $C_{2}^{v}=O_{2}^{v} \cap P_{2}^{v}$ ). From the two spheres $O_{1}^{u}$ and $O_{2}^{v}$, we can find a plane $P_{12}^{u v}$ that contains the intersection circle $C_{12}^{u v}=O_{1}^{u} \cap O_{2}^{v}$. Then the three planes $P_{1}^{u}, P_{2}^{v}$, and $P_{12}^{u v}$ determine a common intersection point $\mathbf{x}(u, v)$. When we substitute the point equation $\mathbf{x}(u, v)$ into the implicit equation of $O_{1}^{u}$ or $O_{2}^{v}$, we get an equation $\lambda(u, v)=0$ of relatively low 
degree. Thus the intersection problem is reformulated as the problem of finding the zero-set of $\lambda(u, v)=0$. (An efficient and robust algorithm for tracing all branches of the zero-set can be found in a recent survey paper of Patrikalakis and Maekawa [18] and the references cited in the paper.)

The problem-reduction scheme employed in this paper is not limited to the intersection of two ringed surfaces. A similar technique has been used in the intersection of two ruled surfaces [8]. In this paper, we also present an algorithm for the intersection of a ringed surface and a ruled surface. More generally, Kim and Elber [13] reviewed the paradigm that consists of converting various interesting geometric problems to a search for the zero-set of constraint equations in the parameter space of input curves and surfaces. Some applications include the computation of sweeps and Minkowski sums of freeform curves and surfaces. Nonrational bisectors for freeform curves and surfaces have also been computed using the same method of problem reduction [4, 5]. Elber and Kim [6] present an efficient algorithm that solves multivariate rational equations, the bivariate case of which was used in the implementation of algorithms presented in this paper.

Among all these applications, the intersection of two ringed surfaces is the most interesting, because at first it looks to be a nonlinear problem, whereas other problems have rather obvious linear structures in their constraint equations. The construction of three planes in a general position is the key idea for reducing this intersection problem to one of finding a zero-set in the parameter space of the two original ringed surfaces.

Computing the self-intersection of a freeform surface is usually more difficult than the case of intersecting two different surfaces. Two identical surfaces are tangential everywhere; thus, a subdivision-based approach would not work for this case. On the other hand, our algorithm can deal with the self-intersection of a ringed surface quite easily, with a slight modification to the original algorithm. Let $\cup_{u} C^{u}$ and $\cup_{v} C^{v}$ be two different parameterizations of the same ringed surface. Clearly, $u-v=0$ is a trivial solution of $\lambda(u, v)=0$ since $C^{u}$ and $C^{v}$ represent the same circle when $u=v$. This paper shows that the zero-set of $\bar{\lambda}(u, v)=\lambda(u, v) /(u-v)^{4}=0$ corresponds to the self-intersection of a ringed surface.

Maekawa et al. [14] suggested a method of designing a pipe surface (a special case of a ringed surface) that has no local or global self-intersection. Though the algorithm does not construct the intersection curve explicitly, the intersection test between two end circles (of the same radius) also uses the principle of three planes, where the third plane $P_{12}^{u v}$ is now the bisector plane between the centers of the two circles.

Surfaces of linear extrusion and surfaces of revolution are among the most frequently used surfaces in designing simple three-dimensional shapes. They belong to the class of ruled and ringed surfaces. Using the result of this paper and also that of Heo et al. [8] for intersecting two ruled surfaces, we can deal with the cases of intersecting surfaces of linear extrusion and surfaces of revolution. In addition to that, there is a more efficient way of computing the intersection of these surfaces since they provide some special structures that we can utilize for speeding up the computation of the zero-set $\lambda(u, v)=0$. For example, in the case of intersecting two surfaces of linear extrusion, the bivariate equation can be decomposed in the form of $\lambda(u, v)=f(u)-g(v)=0$. Moreover, in the case of intersecting two surfaces of revolution, the zero-set of $\lambda(u, v)=0$ can be reduced to $\|\mathbf{f}(u)-\mathbf{g}(v)\|=|r(u)|$, where $\mathbf{f}(u)$ and $\mathbf{g}(v)$ are space curves and $r(u)$ is a scalar function. In the case of intersecting a surface of revolution and a surface of linear extrusion, the space curve $\mathbf{f}(u)$ reduces to a line $\mathbf{l}(u)$, which can further simplify the zero-set computation. 
The rest of this paper is organized as follows. In Section 2, the intersection of two ringed surfaces is reduced to the problem of computing the zero-set of a bivariate function $\lambda(u, v)=0$. Redundant solutions from this zero-set approach are discussed in Section 3, and degenerate cases are analyzed in Section 4 . Section 5 introduces an algorithm to compute the self-intersection of a ringed surface. Section 6 presents an algorithm for the intersection of a ringed surface and a ruled surface, and Section 7 considers the intersection of two simple sweep surfaces such as surfaces of revolution and surfaces of linear extrusion. Finally, Section 8 concludes this paper.

\section{PROBLEM REDUCTION}

Let $S_{1}(u, s)$ and $S_{2}(v, t)$ be two ringed surfaces, each of which is defined as the union of a one-parameter family of circles

$$
S_{1}(u, s)=\cup_{u} C_{1}^{u}(s) \quad \text { and } \quad S_{2}(v, t)=\cup_{v} C_{2}^{v}(t),
$$

where $C_{1}^{u}(s)$ and $C_{2}^{v}(t)$ are circles parameterized by $s$ and $t$, respectively. Assume that the circle $C_{1}^{u}$ has center $\mathbf{p}_{1}(u)=\left(p_{1, x}(u), p_{1, y}(u), p_{1, z}(u)\right)$ and radius $r_{1}(u)$ and, moreover, that $C_{1}^{u}$ is contained in the plane $P_{1}^{u}$ with normal $\mathbf{n}_{1}(u)=\left(n_{1, x}(u), n_{1, y}(u), n_{1, z}(u)\right)$. Let $O_{1}^{u}$ denote a sphere with center $\mathbf{p}_{1}(u)$ and radius $r_{1}(u)$. Then we have

$$
C_{1}^{u}=P_{1}^{u} \cap O_{1}^{u}
$$

Similarly, the circle $C_{2}^{v}$ and sphere $O_{2}^{v}$ have their center $\mathbf{p}_{2}(v)=\left(p_{2, x}(v), p_{2, y}(v), p_{2, z}(v)\right)$ and radius $r_{2}(v)$; moreover, the circle $C_{2}^{v}$ is contained in the plane $P_{2}^{v}$ with normal $\mathbf{n}_{2}(v)=$ $\left(n_{2, x}(v), n_{2, y}(v), n_{2, z}(v)\right)$, and so we find that

$$
C_{2}^{v}=P_{2}^{v} \cap O_{2}^{v}
$$

Thus the intersection $S_{1} \cap S_{2}$ of our ringed surfaces is shown to be the union of a twoparameter family of circle-circle intersections

$$
\begin{aligned}
S_{1} \cap S_{2} & =\cup_{u} \cup_{v} C_{1}^{u} \cap C_{2}^{v} \\
& =\cup_{u} \cup_{v}\left(P_{1}^{u} \cap O_{1}^{u}\right) \cap\left(P_{2}^{v} \cap O_{2}^{v}\right) \\
& =\cup_{u} \cup_{v}\left(P_{1}^{u} \cap P_{2}^{v}\right) \cap\left(O_{1}^{u} \cap O_{2}^{v}\right) \\
& =\cup_{u} \cup_{v}\left(P_{1}^{u} \cap P_{2}^{v}\right) \cap C_{12}^{u v} \\
& =\cup_{u} \cup_{v}\left(P_{1}^{u} \cap P_{2}^{v}\right) \cap\left(P_{12}^{u v} \cap O_{1}^{u}\right) \\
& =\cup_{u} \cup_{v}\left(P_{1}^{u} \cap P_{2}^{v} \cap P_{12}^{u v}\right) \cap O_{1}^{u} \\
& =\cup_{u} \cup_{v}\left(P_{1}^{u} \cap P_{2}^{v}\right) \cap\left(P_{12}^{u v} \cap O_{2}^{v}\right) \\
& =\cup_{u} \cup_{v}\left(P_{1}^{u} \cap P_{2}^{v} \cap P_{12}^{u v}\right) \cap O_{2}^{v},
\end{aligned}
$$

where $C_{12}^{u v}$ is the circle formed by the intersection of the two spheres $O_{1}^{u}$ and $O_{2}^{v}$, and $P_{12}^{u v}$ is the plane containing the circle $C_{12}^{u v}$.

A simple calculation shows that the plane $P_{12}^{u v}$ contains the following point:

$$
\frac{\mathbf{p}_{1}(u)+\mathbf{p}_{2}(v)}{2}+\frac{r_{1}^{2}(u)-r_{2}^{2}(v)}{2\left\|\mathbf{p}_{1}(u)-\mathbf{p}_{2}(v)\right\|^{2}}\left(\mathbf{p}_{2}(v)-\mathbf{p}_{1}(u)\right) .
$$


Moreover, the difference vector $\mathbf{p}_{1}(u)-\mathbf{p}_{2}(v)$ is normal to the plane $P_{12}^{u v}$. Note that the inner product of this normal vector and the position vector of Eq. (1) produce the scalar quantity

$$
\frac{\left\|\mathbf{p}_{1}(u)\right\|^{2}-\left\|\mathbf{p}_{2}(v)\right\|^{2}+r_{2}^{2}(u)-r_{1}^{2}(v)}{2} .
$$

The intersection point $(\hat{x}(u, v), \hat{y}(u, v), \hat{z}(u, v))$ of the three planes $P_{1}^{u}, P_{2}^{v}$, and $P_{12}^{u v}$ satisfies the following matrix equation:

$$
\left[\begin{array}{c}
\mathbf{n}_{1}(u) \\
\mathbf{n}_{2}(v) \\
\mathbf{p}_{1}(u)-\mathbf{p}_{2}(v)
\end{array}\right]\left[\begin{array}{c}
\hat{x}(u, v) \\
\hat{y}(u, v) \\
\hat{z}(u, v)
\end{array}\right]=\left[\begin{array}{c}
\left\langle\mathbf{n}_{1}(u), \mathbf{p}_{1}(u)\right\rangle \\
\left\langle\mathbf{n}_{2}(v), \mathbf{p}_{2}(v)\right\rangle \\
\frac{\left\|\mathbf{p}_{1}(u)\right\|^{2}-\left\|\mathbf{p}_{2}(v)\right\|^{2}+r_{2}^{2}(v)-r_{1}^{2}(u)}{2}
\end{array}\right] .
$$

When the three vectors $\mathbf{n}_{1}(u), \mathbf{n}_{2}(v)$, and $\mathbf{p}_{1}(u)-\mathbf{p}_{2}(v)$ are linearly independent, the above equation has a unique solution $(\hat{x}(u, v), \hat{y}(u, v), \hat{z}(u, v))$.

We now define bivariate functions $w(u, v), x(u, v), y(u, v), z(u, v)$ as follows:

$$
\begin{aligned}
w(u, v) & =\left|\begin{array}{c}
\mathbf{n}_{1}(u) \\
\mathbf{n}_{2}(v) \\
\mathbf{p}_{1}(u)-\mathbf{p}_{2}(v)
\end{array}\right|, \\
x(u, v) & =\left|\begin{array}{ccc}
\left\langle\mathbf{n}_{1}(u), \mathbf{p}_{1}(u)\right\rangle & n_{1, y}(u) & n_{1, z}(u) \\
\left\langle\mathbf{n}_{2}(v), \mathbf{p}_{2}(v)\right\rangle & n_{2, y}(v) & n_{2, z}(v) \\
\mu(u, v) & p_{12, y}(u, v) & p_{12, z}(u, v)
\end{array}\right|, \\
y(u, v) & =\left|\begin{array}{ccc}
n_{1, x}(u) & \left\langle\mathbf{n}_{1}(u), \mathbf{p}_{1}(u)\right\rangle & n_{1, z}(u) \\
n_{2, x}(v) & \left\langle\mathbf{n}_{2}(v), \mathbf{p}_{2}(v)\right\rangle & n_{2, z}(v) \\
p_{12, x}(u, v) & \mu(u, v) & p_{12, z}(u, v)
\end{array}\right|, \\
z(u, v) & =\left|\begin{array}{ccc}
n_{1, x}(u) & n_{1, y}(u) & \left\langle\mathbf{n}_{1}(u), \mathbf{p}_{1}(u)\right\rangle \\
n_{2, x}(v) & n_{2, y}(v) & \left\langle\mathbf{n}_{2}(v), \mathbf{p}_{2}(v)\right\rangle \\
p_{12, x}(u, v) & p_{12, y}(u, v) & \mu(u, v)
\end{array}\right|,
\end{aligned}
$$

where

$$
\mu(u, v)=\frac{\left\|\mathbf{p}_{1}(u)\right\|^{2}-\left\|\mathbf{p}_{2}(v)\right\|^{2}+r_{2}^{2}(v)-r_{1}^{2}(u)}{2}
$$

and

$$
\begin{aligned}
& p_{12, x}(u, v)=p_{1, x}(u)-p_{2, x}(v), \\
& p_{12, y}(u, v)=p_{1, y}(u)-p_{2, y}(v), \\
& p_{12, z}(u, v)=p_{1, z}(u)-p_{2, z}(v) .
\end{aligned}
$$

By Cramer's rule, the intersection point $(\hat{x}(u, v), \hat{y}(u, v), \hat{z}(u, v))$ can then be computed from

$$
\hat{x}(u, v)=\frac{x(u, v)}{w(u, v)}, \quad \hat{y}(u, v)=\frac{y(u, v)}{w(u, v)}, \quad \hat{z}(u, v)=\frac{z(u, v)}{w(u, v)} .
$$




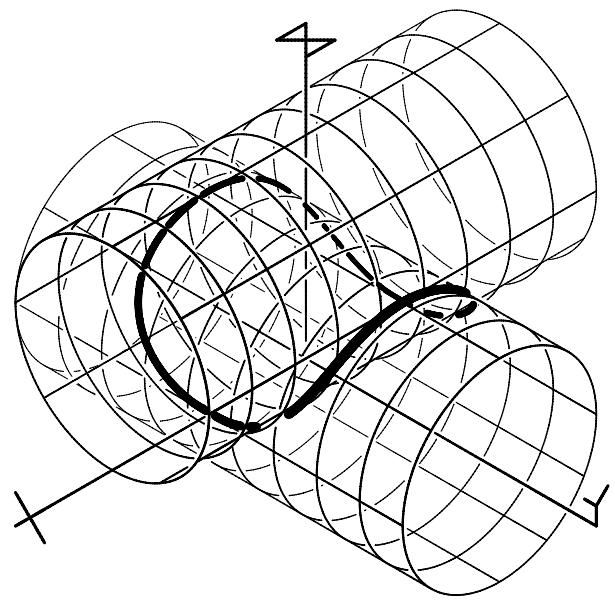

(a)

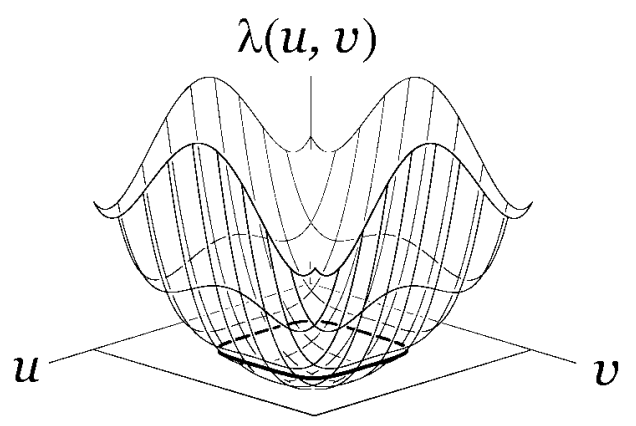

(b)

FIG. 1. The intersection of two cylinders: (a) two cylinders and (b) $\lambda$-function.

The bivariate functions $w(u, v), x(u, v), y(u, v)$, and $z(u, v)$ are polynomial if the position curves $\mathbf{p}_{1}(u)$ and $\mathbf{p}_{2}(v)$, the radius functions $r_{1}(u)$ and $r_{2}(v)$, and the normal vectors $\mathbf{n}_{1}(u)$ and $\mathbf{n}_{2}(v)$ are all polynomial; if the position curves, radius functions, and normal vectors are rational, the bivariate functions are rational. The condition for the intersection point $\left(\frac{x(u, v)}{w(u, v)}, \frac{y(u, v)}{w(u, v)}, \frac{z(u, v)}{w(u, v)}\right)$ to be located on the sphere $O_{1}^{u}$ can be formulated as follows:

$$
\lambda(u, v)=\left\|(x(u, v), y(u, v), z(u, v))-w(u, v) \mathbf{p}_{1}(u)\right\|^{2}-w^{2}(u, v) r_{1}^{2}(u)=0 .
$$

When the vectors $\mathbf{n}_{1}(u), \mathbf{n}_{2}(v)$, and $\mathbf{p}_{1}(u)-\mathbf{p}_{2}(v)$ are linearly independent, the equation $\lambda(u, v)=0$ is a necessary and sufficient condition for two circles $C_{1}^{u}$ and $C_{2}^{v}$ to have a nonempty intersection point. For each point $(u, v)$ in the zero-set of $\lambda(u, v)=0$, the corresponding intersection point can be computed by the rational map $\left(\frac{x(u, v)}{w(u, v)}, \frac{y(u, v)}{w(u, v)}, \frac{z(u, v)}{w(u, v)}\right)$, which is the solution of Eq. (2). Figures 1 and 2 show some examples of intersecting two ringed surfaces.

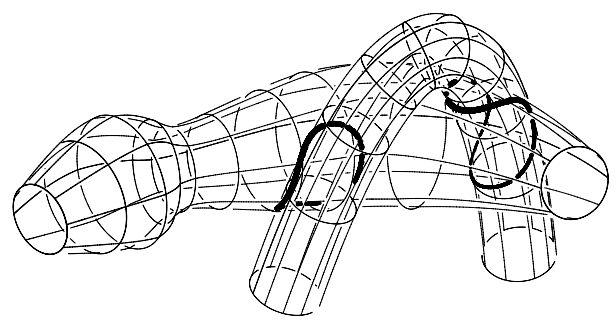

(a)

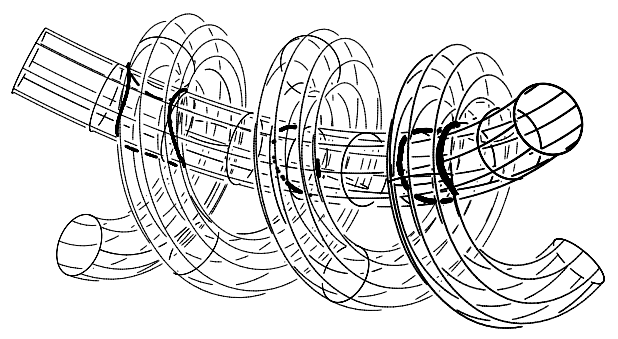

(b)

FIG. 2. Intersecting two ringed surfaces. 
EXAMPLE 1. Let two ringed surfaces $S_{1}(u, s)=\cup_{u} C_{1}^{u}$ and $S_{2}(v, t)=\cup_{v} C_{2}^{v}$ be defined by the following functions:

$$
\begin{array}{lll}
\mathbf{p}_{1}(u)=(0,4 u-2,0), & r_{1}(u)=1, & \mathbf{n}_{1}(u)=(0,1,0), \\
\mathbf{p}_{2}(v)=(4 v-2,0,1), & r_{2}(v)=1, & \mathbf{n}_{2}(v)=(1,0,0) .
\end{array}
$$

Note that $S_{1}(u, s)$ and $S_{2}(v, t)$ are two cylinders intersecting orthogonally (see Fig. 1a).

From these definitions we can formulate the matrix equation

$$
\left[\begin{array}{ccc}
0 & 1 & 0 \\
1 & 0 & 0 \\
2-4 v & 4 u-2 & -1
\end{array}\right]\left[\begin{array}{c}
\frac{x(u, v)}{w(u, v)} \\
\frac{y(u, v)}{w(u, v)} \\
\frac{z(u, v)}{w(u, v)}
\end{array}\right]=\left[\begin{array}{c}
4 u-2 \\
4 v-2 \\
8 u^{2}-8 u-8 v^{2}+8 v-\frac{1}{2}
\end{array}\right]
$$

where the bivariate functions $w(u, v), x(u, v), y(u, v)$, and $z(u, v)$ are defined as follows

$$
\begin{aligned}
& w(u, v)=1, \\
& x(u, v)=4 v-2, \\
& y(u, v)=4 u-2, \\
& z(u, v)=8 u^{2}-8 u-8 v^{2}+8 v+\frac{1}{2} .
\end{aligned}
$$

Finally, the bivariate function $\lambda(u, v)$ may be computed by means of the equation:

$$
\lambda(u, v)=(4 v-2)^{2}+\left(8 u^{2}-8 u-8 v^{2}+8 v+\frac{1}{2}\right)^{2}-1=0
$$

From the zero-set of $\lambda(u, v)=0$, we can now construct the intersection curve $\left(\frac{x(u, v)}{w(u, v)}, \frac{y(u, v)}{w(u, v)}\right.$, $\left.\frac{z(u, v)}{w(u, v)}\right)$ (see Fig. 1).

\section{REDUNDANT SOLUTIONS}

When the three vectors $\mathbf{n}_{1}(u), \mathbf{n}_{2}(v)$, and $\mathbf{p}_{1}(u)-\mathbf{p}_{2}(v)$ are linearly dependent, we have

$$
w(u, v)=\left\langle\mathbf{n}_{1}(u) \times \mathbf{n}_{2}(v), \mathbf{p}_{1}(u)-\mathbf{p}_{2}(v)\right\rangle=0 .
$$

Thus the condition $\lambda(u, v)=0$ reduces to

$$
\begin{aligned}
\lambda(u, v) & =\left\|(x(u, v), y(u, v), z(u, v))-w(u, v) \mathbf{p}_{1}(u)\right\|^{2}-w^{2}(u, v) r_{1}^{2}(u) \\
& =\|(x(u, v), y(u, v), z(u, v))\|^{2} \\
& =0
\end{aligned}
$$

which is equivalent to

$$
x(u, v)=y(u, v)=z(u, v)=0 .
$$


As we discuss below, when $w(u, v)=0$, there are some redundant cases where $\lambda(u, v)=0$ holds but the two circles $C_{1}^{u}$ and $C_{2}^{v}$ have no intersection. Consequently, when the three vectors $\mathbf{n}_{1}(u), \mathbf{n}_{2}(v)$, and $\mathbf{p}_{1}(u)-\mathbf{p}_{2}(v)$ are linearly dependent, $\lambda(u, v)=0$ is only a necessary, but not a sufficient, condition for the two circles $C_{1}^{u}$ and $C_{2}^{v}$ to have intersection at a real affine point.

The condition $w(u, v)=x(u, v)=y(u, v)=z(u, v)=0$ means that the three planes $P_{1}^{u}, P_{2}^{v}$, and $P_{12}^{u v}$ meet either at infinitely many real affine points or at points at infinity; thus the following two cases are the only ones that we need to consider for intersection at real affine points:

1. The two planes $P_{1}^{u}$ and $P_{2}^{v}$ are coplanar.

2. The three planes $P_{1}^{u}, P_{2}^{v}$, and $P_{12}^{u v}$ intersect in a line.

The first case can be classified by the following two equations:

$$
\begin{aligned}
\Delta(u, v) & =\left\|\mathbf{n}_{1}(u) \times \mathbf{n}_{2}(v)\right\|^{2}=0, \\
\delta(u, v) & =\left\langle\mathbf{n}_{1}(u), \mathbf{p}_{1}(u)-\mathbf{p}_{2}(v)\right\rangle=0 .
\end{aligned}
$$

The circle-circle intersection problem is then essentially reduced to the planar case.

When the two planes $P_{1}^{u}$ and $P_{2}^{v}$ are identical and when, additionally, $\mathbf{p}_{1}(u)=\mathbf{p}_{2}(v)$, the two circles $C_{1}^{u}$ and $C_{2}^{v}$ intersect if and only if $r_{1}^{2}(u)=r_{2}^{2}(v)$; in this case, the two circles are identical.

When the two planes $P_{1}^{u}$ and $P_{2}^{v}$ overlap and $\mathbf{p}_{1}(u) \neq \mathbf{p}_{2}(v)$, the third plane $P_{12}^{u v}$ is orthogonal to both $P_{1}^{u}$ and $P_{2}^{v}$. The circle-circle intersection points are then located on the intersection line $P_{1}^{u} \cap P_{12}^{u v}$ of two planes. This line is parallel to $\mathbf{n}_{1}(u) \times\left(\mathbf{p}_{1}(u)-\mathbf{p}_{2}(v)\right)$, and it contains the point

$$
\mathbf{m}(u, v)=\frac{\mathbf{p}_{1}(u)+\mathbf{p}_{2}(v)}{2}+\frac{r_{1}^{2}(u)-r_{2}^{2}(v)}{2\left\|\mathbf{p}_{1}(u)-\mathbf{p}_{2}(v)\right\|^{2}}\left(\mathbf{p}_{2}(v)-\mathbf{p}_{1}(u)\right),
$$

which is also the foot point of the perpendicular from $\mathbf{p}_{1}(u)$ to the intersection line. The two intersection points $C_{1}^{u} \cap C_{2}^{v}$ are computed as follows

$$
\mathbf{m}(u, v) \pm \sqrt{r_{1}^{2}(u)-\left\|\mathbf{m}(u, v)-\mathbf{p}_{1}(u)\right\|^{2}} \cdot \frac{\mathbf{n}_{1}(u) \times\left(\mathbf{p}_{1}(u)-\mathbf{p}_{2}(v)\right)}{\left\|\mathbf{n}_{1}(u) \times\left(\mathbf{p}_{1}(u)-\mathbf{p}_{2}(v)\right)\right\|} .
$$

Now, when the three planes $P_{1}^{u}, P_{2}^{v}$, and $P_{12}^{u v}$ intersect in a real affine line, but $P_{1}^{u}$ and $P_{2}^{v}$ are not parallel, we have

$$
\lambda(u, v)=0, \quad w(u, v)=0, \quad \text { and } \quad \Delta(u, v)>0 .
$$

The common intersection line (parameterized by $t$ ) can be written

$$
L^{u v}(t)=\mathbf{m}(u, v)+t \mathbf{n}_{1}(u) \times \mathbf{n}_{2}(v),
$$

where $\mathbf{m}(u, v)$ is the foot point of the perpendicular from $\mathbf{p}_{1}(u)$ to the line. Note that the foot point $\mathbf{m}(u, v)$ satisfies the following equations:

$$
\begin{aligned}
\left\langle\mathbf{n}_{1}(u), \mathbf{m}(u, v)-\mathbf{p}_{1}(u)\right\rangle & =0, \\
\left\langle\mathbf{n}_{2}(v), \mathbf{m}(u, v)-\mathbf{p}_{2}(v)\right\rangle & =0, \\
\left\langle\mathbf{n}_{1}(u) \times \mathbf{n}_{2}(v), \mathbf{m}(u, v)-\mathbf{p}_{1}(u)\right\rangle & =0 .
\end{aligned}
$$


Since the three vectors $\mathbf{n}_{1}(u), \mathbf{n}_{2}(v)$, and $\mathbf{n}_{1}(u) \times \mathbf{n}_{2}(v)$ are linearly independent, the foot point $\mathbf{m}(u, v)$ has a rational representation in $u$ and $v$.

The two circles $C_{1}^{u}$ and $C_{2}^{v}$ intersect in two real affine points if and only if

$$
r_{1}^{2}(u)-\left\|\mathbf{m}(u, v)-\mathbf{p}_{1}(u)\right\|^{2}=r_{2}^{2}(v)-\left\|\mathbf{m}(u, v)-\mathbf{p}_{2}(v)\right\|^{2}
$$

And the two intersection points $C_{1}^{u} \cap C_{2}^{v}$ are then computed as

$$
\mathbf{m}(u, v) \pm \sqrt{r_{1}^{2}(u)-\left\|\mathbf{m}(u, v)-\mathbf{p}_{1}(u)\right\|^{2}} \cdot \frac{\mathbf{n}_{1}(u) \times \mathbf{n}_{2}(v)}{\left\|\mathbf{n}_{1}(u) \times \mathbf{n}_{2}(v)\right\|}
$$

\section{DEGENERATE CASES}

In general, the solution-set of $\lambda(u, v)=0$ corresponds to a planar algebraic curve in the $u v$-plane. However, in some degenerate cases, the solution-set may contain the whole $u v$-plane (i.e., $\lambda(u, v) \equiv 0)$. When each circle $C_{1}^{u}$ of the first ringed surface $S_{1}$ intersects all the circles $C_{2}^{v}$ of the second ringed surface $S_{2}$, we have $\lambda(u, v) \equiv 0$.

For example, the torus may be represented as a union of cross-sectional circles; at the same time, it can also be represented as a union of profile circles [11]. (There are two more ways of representing the torus as a union of circles, i.e., as a union of Yvone-Villarceau circles.) When we intersect the two representations of a single torus with each other, each cross-sectional circle on one torus intersects all profile circles on the other, and vice versa. Consequently, the resulting $\lambda$-function vanishes identically: $\lambda(u, v) \equiv 0$. Similarly, there are four different ways of decomposing a Dupin cyclide into a union of circles: we can take either of the original surfaces as the intersection of the two ringed surfaces.

Consider a ringed surface with a singular point where all the circles intersect. (A spindle torus, for example, has two such singular points.) When we place two ringed surfaces of this type so that their respective singular points share the same position, each circle from one ringed surface will intersect all the circles on the other ringed surface, and vice versa. Consequently, $\lambda(u, v) \equiv 0$.

Two ringed surfaces may intersect at some points in addition to the singular point. The zero-set of $\lambda(u, v) \equiv 0$ is of no use for the construction of these intersection points, other than the singular point. When two circles $C_{1}^{u}$ and $C_{2}^{v}$ intersect at a point other than the singular point, the three planes $P_{1}^{u}, P_{2}^{v}$, and $P_{12}^{u v}$ intersect in a straight line through the intersection point and the singular point. Thus we can apply the techniques introduced in the previous section to such degenerate cases.

There are some other degenerate cases where the three normal vectors $\mathbf{n}_{1}(u), \mathbf{n}_{2}(v)$, and $\mathbf{p}_{1}(u)-\mathbf{p}_{2}(v)$ are linearly dependent for all $(u, v)$ : that is, cases where $\lambda(u, v) \equiv 0$ and $w(u, v) \equiv 0$, and consequently $x(u, v) \equiv y(u, v) \equiv z(u, v) \equiv 0$. As we have discussed in the previous section, there are essentially two cases to consider:

1. The two planes $P_{1}^{u}$ and $P_{2}^{v}$ overlap, for all $u$ and $v$.

2. The three planes $P_{1}^{u}, P_{2}^{v}$, and $P_{12}^{u v}$ intersect in a line, for all $u$ and $v$.

It is easy to detect the first case, where the two ringed surfaces degenerate to planar regions contained in the same plane. The second case is more interesting. An example includes the case of intersecting two tori with the same axis of rotation and the same major circle, but with different minor radii. 
The detection of degenerate cases involves checking whether certain bivariate functions vanish identically:

$$
F(u, v) \equiv 0
$$

Assume that $F(u, v)$ is a polynomial function of degree $(m, n)$ and that a uniform sampling is made on the $(u, v)$-parameter domain: $\left(u_{i}, v_{j}\right)$, for $i=0, \ldots, m$ and $j=0, \ldots, n$. When we have $F\left(u_{i}, v_{j}\right)=0$ at these $(m+1)(n+1)$ parameter values, we can guarantee that $F(u, v) \equiv 0$. Elber and Kim [3] used similar tests for the shape recognition of freeform curves and surfaces of special types such as surfaces of revolution, surfaces of linear extrusion, ruled surfaces, and developable surfaces.

\section{SELF-INTERSECTION OF A RINGED SURFACE}

Assume that a ringed surface $S(u, t)=\cup_{u} C^{u}(t)$ is defined by the center $\mathbf{p}(u)$ and radius $r(u)$ of the circle $C^{u}$ and by the normal $\mathbf{n}(u)$ of the plane $P^{u}$ that contains the circle $C^{u}$. Then $S(u, t)$ has a self-intersection if and only if $C^{u} \cap C^{v} \neq \emptyset$, for $u \neq v$. Assuming further that the two circles $C^{u}$ and $C^{v}$ intersect at a real affine point $(\hat{x}(u, v), \hat{y}(u, v), \hat{z}(u, v))$, we obtain the matrix equation

$$
\left[\begin{array}{c}
\mathbf{n}(u) \\
\mathbf{n}(v) \\
\mathbf{p}(u)-\mathbf{p}(v)
\end{array}\right]\left[\begin{array}{c}
\hat{x}(u, v) \\
\hat{y}(u, v) \\
\hat{z}(u, v)
\end{array}\right]=\left[\begin{array}{c}
\langle\mathbf{n}(u), \mathbf{p}(u)\rangle \\
\langle\mathbf{n}(v), \mathbf{p}(v)\rangle \\
\frac{\|\mathbf{p}(u)\|^{2}-\|\mathbf{p}(v)\|^{2}+r^{2}(v)-r^{2}(u)}{2}
\end{array}\right],
$$

which is equivalent to

$$
\left[\begin{array}{c}
\mathbf{n}(u) \\
\mathbf{n}(u)-\mathbf{n}(v) \\
\mathbf{p}(u)-\mathbf{p}(v)
\end{array}\right]\left[\begin{array}{c}
\hat{x}(u, v) \\
\hat{y}(u, v) \\
\hat{z}(u, v)
\end{array}\right]=\left[\begin{array}{c}
\langle\mathbf{n}(u), \mathbf{p}(u)\rangle \\
\langle\mathbf{n}(u), \mathbf{p}(u)\rangle-\langle\mathbf{n}(v), \mathbf{p}(v)\rangle \\
\frac{\|\mathbf{p}(u)\|^{2}-\|\mathbf{p}(v)\|^{2}+r^{2}(v)-r^{2}(u)}{2}
\end{array}\right] .
$$

When the functions $\mathbf{p}(u), \mathbf{n}(u)$, and $r(u)$ are polynomial or rational, each of the terms $\mathbf{n}(u)-\mathbf{n}(v), \mathbf{p}(u)-\mathbf{p}(v),\langle\mathbf{n}(u), \mathbf{p}(u)\rangle-\langle\mathbf{n}(v), \mathbf{p}(v)\rangle$, and $\|\mathbf{p}(u)\|^{2}-\|\mathbf{p}(v)\|^{2}+r^{2}(v)-$ $r^{2}(u)$ has a factor $(u-v)$, and we can deduce that

$$
(u-v)^{2}\left[\begin{array}{c}
\mathbf{n}(u) \\
\frac{\mathbf{n}(u)-\mathbf{n}(v)}{u-v} \\
\frac{\mathbf{p}(u)-\mathbf{p}(v)}{u-v}
\end{array}\right]\left[\begin{array}{c}
\hat{x}(u, v) \\
\hat{y}(u, v) \\
\hat{z}(u, v)
\end{array}\right]=(u-v)^{2}\left[\begin{array}{c}
\langle\mathbf{n}(u), \mathbf{p}(u)\rangle \\
\frac{\langle\mathbf{n}(u), \mathbf{p}(u)\rangle-\langle\mathbf{n}(v), \mathbf{p}(v)\rangle}{u-v} \\
\frac{\|\mathbf{p}(u)\|^{2}-\|\mathbf{p}(v)\|^{2}+r^{2}(v)-r^{2}(u)}{2(u-v)}
\end{array}\right] .
$$

From the above equation, it is clear that the terms $w(u, v), x(u, v), y(u, v)$, and $z(u, v)$ have a factor of $(u-v)^{2}$. Thus the following bivariate function has a factor of $(u-v)^{4}$ :

$$
\lambda(u, v)=\|(x(u, v), y(u, v), z(u, v))-w(u, v) \mathbf{p}(u)\|^{2}-w^{2}(u, v) r^{2}(u) .
$$

Now let us define a simplified function

$$
\bar{\lambda}(u, v)=\frac{\lambda(u, v)}{(u-v)^{4}} .
$$




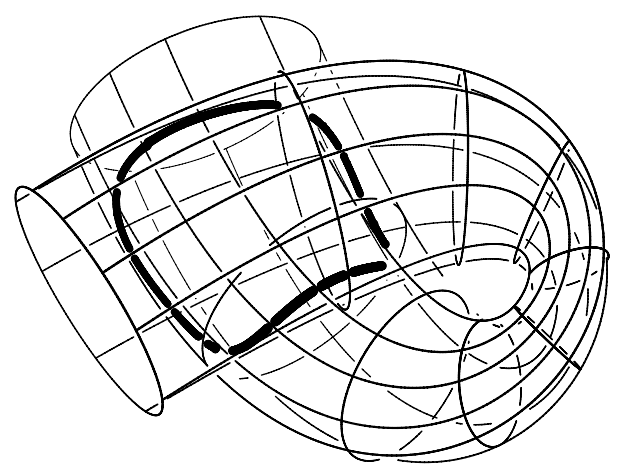

(a)

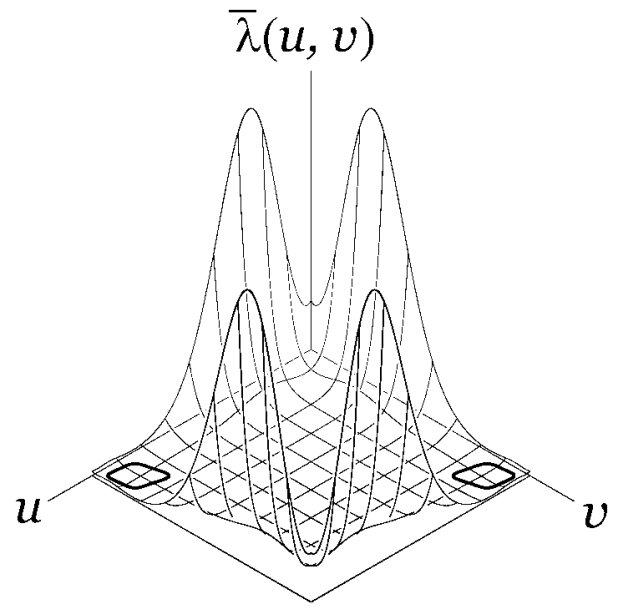

(b)

FIG. 3. A self-intersecting ringed surface: (a) a ringed surface and (b) $\bar{\lambda}(u, v)$.

The zero-set of $\bar{\lambda}(u, v)=0$ is the same as that of $\lambda(u, v)=0$ except the solutions on the diagonal line $u=v$. Moreover, since $\bar{\lambda}(u, v)$ is symmetric with respect to $u$ and $v$, the zero-set of $\bar{\lambda}(u, v)=0$ is also symmetric with respect to the diagonal line $u=v$. Thus we need to compute the zero-set of $\bar{\lambda}(u, v)=0$ only when $u>v$.

EXAMPLE 3. Let a ringed surface $S(u, t)=\cup_{u} C^{u}(t)$ be defined by the following functions

$$
\begin{aligned}
& \mathbf{p}(u)=\sum_{i=0}^{3}\left(\begin{array}{l}
3 \\
i
\end{array}\right) u^{i}(1-u)^{3-i} \mathbf{p}_{i}, \\
& r(u)=\left(u-\frac{1}{2}\right)^{2}+\frac{1}{4}, \\
& \mathbf{n}(u)=\frac{1}{6} \mathbf{p}^{\prime}(u),
\end{aligned}
$$

where $\mathbf{p}_{0}=\left(-\frac{1}{2}, 0,0\right), \mathbf{p}_{1}=\left(2,2, \frac{1}{4}\right), \mathbf{p}_{2}=\left(-2,2, \frac{1}{4}\right)$, and $\mathbf{p}_{3}=\left(\frac{1}{2}, 0, \frac{1}{2}\right)$ are the control points of the cubic Bézier curve $\mathbf{p}(u)$. Figure 3 shows a self-intersecting ringed surface and a $\bar{\lambda}$-function of the ringed surface. Note that the $\bar{\lambda}$-function is symmetric with respect to $u$ and $v$, and there is a connected component of the zero-set in the region $u>v$.

\section{THE INTERSECTION OF A RINGED SURFACE AND A RULED SURFACE}

Let $S_{1}(u, s)$ be a ringed surface defined by

$$
S_{1}(u, s)=\cup_{u} C^{u}(s)
$$

where the circle $C^{u}$ has center $\mathbf{p}(u)=\left(p_{x}(u), p_{y}(u), p_{z}(u)\right)$ and radius $r(u)$ and, moreover, that $C^{u}$ is contained in the plane $P^{u}$ with normal $\mathbf{n}(u)=\left(n_{x}(u), n_{y}(u), n_{z}(u)\right)$. Let $O^{u}$ 
denote a sphere with center $\mathbf{p}(u)$ and radius $r(u)$. Then we have

$$
C^{u}=P^{u} \cap O^{u}
$$

Now, let $S_{2}(v, t)$ be a ruled surface defined by

$$
S_{2}(v, t)=\mathbf{q}(v)+t \mathbf{d}(v),
$$

where $\mathbf{q}(v)$ is a generating curve and $\mathbf{d}(v) \neq 0$ is a direction vector of the ruled surface.

When the two surfaces $S_{1}(u, s)$ and $S_{2}(v, t)$ intersect, the ruling line $\mathbf{q}(v)+t \mathbf{d}(v)$, parameterized by $t$, intersects the plane $P^{u}$;

$$
\langle\mathbf{n}(u), \mathbf{q}(v)+t \mathbf{d}(v)-\mathbf{p}(u)\rangle=0,
$$

which produces the parameter value of $t$ at the intersection point:

$$
t(u, v)=\frac{\langle\mathbf{n}(u), \mathbf{p}(u)-\mathbf{q}(v)\rangle}{\langle\mathbf{n}(u), \mathbf{d}(v)\rangle} .
$$

The intersection point itself is given as

$$
\frac{\langle\mathbf{n}(u), \mathbf{d}(v)\rangle \mathbf{q}(v)+\langle\mathbf{n}(u), \mathbf{p}(u)-\mathbf{q}(v)\rangle \mathbf{d}(v)}{\langle\mathbf{n}(u), \mathbf{d}(v)\rangle} .
$$

When this point is located on the sphere $O^{u}$, we have

$$
\begin{aligned}
\lambda(u, v) \\
=\|\langle\mathbf{n}(u), \mathbf{d}(v)\rangle \mathbf{q}(v)-\langle\mathbf{n}(u), \mathbf{q}(v)\rangle \mathbf{d}(v)+\langle\mathbf{n}(u), \mathbf{p}(u)\rangle \mathbf{d}(v)-\langle\mathbf{n}(u), \mathbf{d}(v)\rangle \mathbf{p}(u)\|^{2} \\
\quad-\langle\mathbf{n}(u), \mathbf{d}(v)\rangle^{2} r^{2}(u) \\
=0 .
\end{aligned}
$$

There are some redundant solutions of $\lambda(u, v)=0$ which do not correspond to real affine intersection points. For example, when the line $\mathbf{q}(v)+t \mathbf{d}(v)$ is totally contained in the plane $P^{u}$ but does not intersect the circle $C^{u}$, there is no real affine intersection point even though the condition $\lambda(u, v)=0$ is satisfied.

Cylinders and cones have dual representation as ruled surfaces as well as ringed surfaces. When we intersect the two different representations of the same surface, a degenerate case occurs, where $\lambda(u, v) \equiv 0$. There are also some other degenerate cases, a complete classification of which will be discussed in Seong et al. [20].

An efficient algorithm for intersecting a ringed surface and a ruled surface can be used for the ray-tracing of ringed surfaces [2]. Figure 4 shows an example of intersecting a Utah teapot (approximated by four ringed surfaces) and a ruled surface (representing a set of reflected rays). Rendering applications involve many tangential intersections, as a result of rays passing through the silhouette of ringed surfaces. It is a challenge to develop an efficient algorithm that can deal with a large number of tangential intersections; we will investigate this direction of research in Seong et al. [20]. 


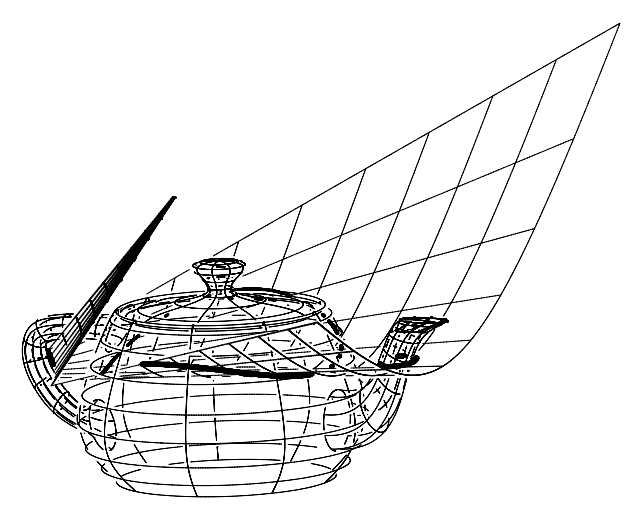

FIG. 4. The intersection of a Utah teapot and a ruled surface of reflected rays.

\section{THE INTERSECTION OF TWO SIMPLE SWEEP SURFACES}

Surfaces of linear extrusion and surfaces of revolution belong to the class of ruled and ringed surfaces. There is a more efficient way of computing the intersection of these simple sweep surfaces since they provide some special structures that we can utilize for speeding up the computation of the zero-set $\lambda(u, v)=0$. This section summarizes the analysis reported in Kim [12]:

1. In the case of intersecting two surfaces of linear extrusion, the bivariate equation can be decomposed in the form of $\lambda(u, v)=f(u)-g(v)=0$.

2. In the case of intersecting two surfaces of revolution, the zero-set of $\lambda(u, v)=0$ can be reduced to $\|\mathbf{f}(u)-\mathbf{g}(v)\|=|r(u)|$, where $\mathbf{f}(u)$ and $\mathbf{g}(v)$ are space curves and $r(u)$ is a scalar function.

3. In the case of intersecting a surface of revolution and a surface of linear extrusion, the space curve $\mathbf{f}(u)$ reduces to a line $\mathbf{l}(u)$, which can further simplify the zero-set computation.

Using the result of Heo et al. [8], we may reformulate the intersection of two ruled surfaces as a zero-set finding problem for a bivariate equation

$$
\lambda(u, v)=\left\langle\mathbf{d}_{1}(u) \times \mathbf{d}_{2}(v), \mathbf{p}_{1}(u)-\mathbf{p}_{2}(v)\right\rangle=0,
$$

where $\mathbf{p}_{1}(u)$ and $\mathbf{p}_{2}(v)$ are generating curves and $\mathbf{d}_{1}(u)$ and $\mathbf{d}_{2}(v)$ are direction vectors of the two ruled surfaces. Surfaces of linear extrusion have fixed direction vectors $\mathbf{d}_{1}$ and $\mathbf{d}_{2}$; thus the bivariate equation can be represented in a decomposable form

$$
\lambda(u, v)=f(u)-g(v)=0,
$$

where

$$
f(u)=\left\langle\mathbf{d}_{1} \times \mathbf{d}_{2}, \mathbf{p}_{1}(u)\right\rangle \quad \text { and } \quad g(v)=\left\langle\mathbf{d}_{1} \times \mathbf{d}_{2}, \mathbf{p}_{2}(v)\right\rangle .
$$

Figure 5 shows an example of intersecting two surfaces of linear extrusion.

Now we consider the intersection of two surfaces of revolution, which is a special case of intersecting two ringed surfaces. Let $S_{1}(u, s)=\cup_{u} C_{1}^{u}(s)$ and $S_{2}(v, t)=\cup_{v} C_{2}^{v}(t)$ be two surfaces of revolution; then we may assume that the circle $C_{1}^{u}$ has center $\mathbf{p}_{1}(u)=\mathbf{p}_{1}+u \mathbf{n}_{1}$ 


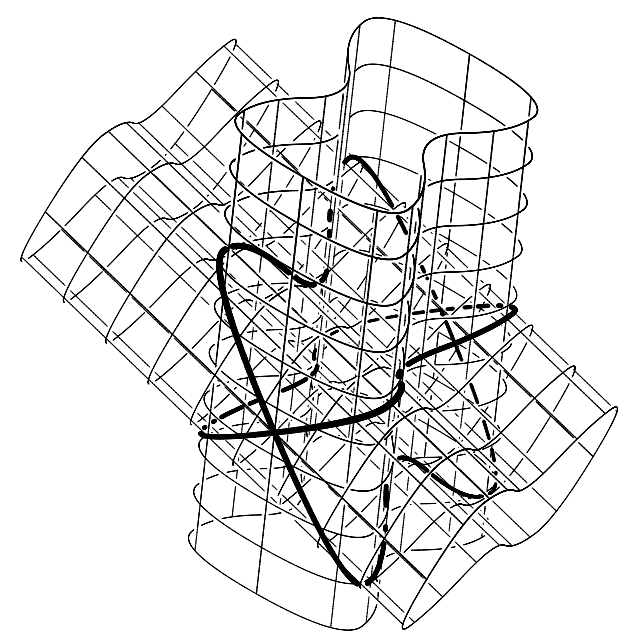

FIG. 5. The intersection of two surfaces of linear extrusion.

and is contained in the plane $P_{1}^{u}$ with a fixed unit normal $\mathbf{n}_{1}$. Similarly, the circle $C_{2}^{v}$ has center $\mathbf{p}_{2}(v)=\mathbf{p}_{2}+v \mathbf{n}_{2}$ and is contained in the plane $P_{2}^{v}$ with a fixed unit normal $\mathbf{n}_{2}$. Equation (2) is then reduced to the matrix equation

$$
\left[\begin{array}{c}
\mathbf{n}_{1} \\
\mathbf{n}_{2} \\
\mathbf{p}_{1}-\mathbf{p}_{2}+u \mathbf{n}_{1}-v \mathbf{n}_{2}
\end{array}\right]\left[\begin{array}{c}
\hat{x}(u, v) \\
\hat{y}(u, v) \\
\hat{z}(u, v)
\end{array}\right]=\left[\begin{array}{c}
\left\langle\mathbf{n}_{1}, \mathbf{p}_{1}\right\rangle+u \\
\left\langle\mathbf{n}_{2}, \mathbf{p}_{2}\right\rangle+v \\
\mu(u, v)
\end{array}\right],
$$

where

$$
\mu(u, v)=\frac{\left\|\mathbf{p}_{1}\right\|^{2}+2 u\left\langle\mathbf{n}_{1}, \mathbf{p}_{1}\right\rangle+u^{2}-\left\|\mathbf{p}_{2}\right\|^{2}-2 v\left\langle\mathbf{n}_{2}, \mathbf{p}_{2}\right\rangle-v^{2}+r_{2}^{2}(v)-r_{1}^{2}(u)}{2} .
$$

By multiplying the first row by $u$ and the second row by $-v$ and subtracting them from the third row of the above matrix equation, we get the following equation:

$$
\left[\begin{array}{c}
\mathbf{n}_{1} \\
\mathbf{n}_{2} \\
\mathbf{p}_{1}-\mathbf{p}_{2}
\end{array}\right]\left[\begin{array}{c}
\hat{x}(u, v) \\
\hat{y}(u, v) \\
\hat{z}(u, v)
\end{array}\right]=\left[\begin{array}{c}
\left\langle\mathbf{n}_{1}, \mathbf{p}_{1}\right\rangle+u \\
\left\langle\mathbf{n}_{2}, \mathbf{p}_{2}\right\rangle+v \\
\frac{\left\|\mathbf{p}_{1}\right\|^{2}-\left\|\mathbf{p}_{2}\right\|^{2}-r_{1}^{2}(u)-u^{2}+r_{2}^{2}(v)+v^{2}}{2}
\end{array}\right] .
$$

Let a constant $w$ be defined as

$$
w=\left|\begin{array}{c}
\mathbf{n}_{1} \\
\mathbf{n}_{2} \\
\mathbf{p}_{1}-\mathbf{p}_{2}
\end{array}\right|=\left\langle\mathbf{n}_{1} \times \mathbf{n}_{2}, \mathbf{p}_{1}-\mathbf{p}_{2}\right\rangle \neq 0
$$

By Cramer's rule, the coordinate function $\hat{x}(u, v)$ is then computed as

$$
\hat{x}(u, v)=w^{-1} \cdot\left|\begin{array}{ccc}
\left\langle\mathbf{n}_{1}, \mathbf{p}_{1}\right\rangle+u & n_{1, y} & n_{1, z} \\
\left\langle\mathbf{n}_{2}, \mathbf{p}_{2}\right\rangle+v & n_{2, y} & n_{2, z} \\
\frac{\left\|\mathbf{p}_{1}\right\|^{2}-\left\|\mathbf{p}_{2}\right\|^{2}-r_{1}^{2}(u)-u^{2}+r_{2}^{2}(v)+v^{2}}{2} & p_{1, y}-p_{2, y} & p_{1, z}-p_{2, z}
\end{array}\right|,
$$




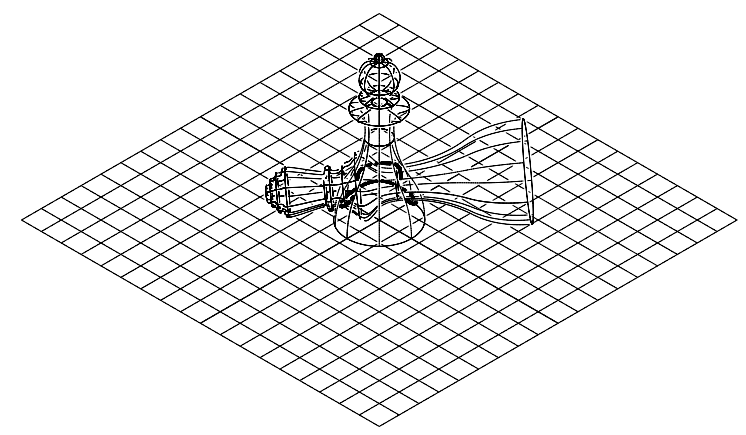

FIG. 6. The intersection of two surfaces of revolution.

where $\mathbf{n}_{i}=\left(n_{i, x}, n_{i, y}, n_{i, z}\right)$ and $\mathbf{p}_{i}=\left(p_{i, x}, p_{i, y}, p_{i, z}\right)$, for $i=1,2$. Note that $\hat{x}(u, v)$ can be represented in the form $f(u)-g(v)$. Similarly, $\hat{y}(u, v)$ and $\hat{z}(u, v)$ can also be represented in this form.

The condition for the intersection point $(\hat{x}(u, v), \hat{y}(u, v), \hat{z}(u, v))$ to be located on the sphere $O_{1}^{u}$ can be formulated as follows

$$
\lambda(u, v)=\left\|(x(u, v), y(u, v), z(u, v))-\mathbf{p}_{1}-u \mathbf{n}_{1}\right\|^{2}-\left|r_{1}(u)\right|^{2}=0 .
$$

Thus the zero-set of $\lambda(u, v)=0$ can be reduced to $\|\mathbf{f}(u)-\mathbf{g}(v)\|=|r(u)|$, where $\mathbf{f}(u)$ and $\mathbf{g}(v)$ are space curves and $r(u)$ is a scalar function. Figure 6 shows an example of intersecting two surfaces of revolution.

In the case of intersecting a surface of revolution and a surface of linear extrusion, the space curve $\mathbf{f}(u)$ reduces to a line $\mathbf{l}(u)$. This can be shown by applying the result of Section 6 to our case. We may assume that $\mathbf{n}(u) \equiv \mathbf{n}, \mathbf{p}(u)=\mathbf{p}+u \mathbf{n}$, and $\mathbf{d}(v) \equiv \mathbf{d}$, for some fixed direction vectors $\mathbf{n}$ and $\mathbf{d}$. Then Eq. (3) of Section 6 is reduced to

$$
\begin{aligned}
\lambda(u, v)= & \|\langle\mathbf{n}, \mathbf{d}\rangle \mathbf{q}(v)-\langle\mathbf{n}, \mathbf{q}(v)\rangle \mathbf{d}+\langle\mathbf{n}, \mathbf{p}+u \mathbf{n}\rangle \mathbf{d}-\langle\mathbf{n}, \mathbf{d}\rangle(\mathbf{p}+u \mathbf{n})\|^{2} \\
& -\langle\mathbf{n}, \mathbf{d}\rangle^{2} r^{2}(u)=0,
\end{aligned}
$$

where the term $\langle\mathbf{n}, \mathbf{p}+u \mathbf{n}\rangle \mathbf{d}-\langle\mathbf{n}, \mathbf{d}\rangle(\mathbf{p}+u \mathbf{n})$ represents a line $\mathbf{l}(u)$.

\section{CONCLUSIONS}

We have presented an efficient and robust algorithm for the intersection of two ringed surfaces and an algorithm for the intersection of a ringed surface and a ruled surface. The intersection problem has been reformulated as the search for the zero-set of a bivariate function. The overall computation procedure is based on the B-spline subdivision technique and is numerically stable [4].

An important advantage of this approach is that the self-intersection of a ringed surface can be similarly reformulated as the zero-set of a bivariate function. Though this paper has not presented the details, the same approach can be applied to the self-intersection of a ruled surface [7].

In the special cases of intersecting surfaces of linear extrusion and surfaces of revolution, the bivariate equations are simplified to certain decomposable forms, $f(u)-g(v)=0$ or 
$\|\mathbf{f}(u)-\mathbf{g}(v)\|=r(u)$, which are considerably easier to compute than the general bivariate equations. We believe that this approach has much potential in accelerating the collision detection among simple sweep surfaces, more details of which will be investigated in future work.

\section{ACKNOWLEDGMENTS}

The authors thank the anonymous referees for their comments which were very useful in improving the presentation of this paper. All the algorithms and figures presented in this paper were implemented and generated using the IRIT solid modeling system [1], developed at the Technion, Israel. This research was supported in part by the Korean Ministry of Information and Communication under the University Basic Research Program of the year 1999, in part by the Korean Ministry of Science and Technology under the National Research Lab Project, and also in part by the Korean Ministry of Education under the Brain Korea 21 Project.

\section{REFERENCES}

1. G. Elber, IRIT 7.0 User's Manual, The Technion-IIT, Haifa, Israel, 1997. Available at http:// www.cs.technion.ac.il/ ${ }^{\sim}$ irit .

2. G. Elber, J. J. Choi, and M.-S. Kim, Ruled tracing, Visual Comput. 13, 1997, 78-94.

3. G. Elber and M.-S. Kim, Geometric shape recognition of freeform curves and surfaces, Graphical Models Image Process. 59, 1997, 417-433.

4. G. Elber and M.-S. Kim, Bisector curves of planar rational curves, Comput. Aided Design 30, 1998, 1089-1096.

5. G. Elber and M.-S. Kim, A computational model for nonrational bisector surfaces: curve-surface and surfacesurface bisectors, in Proc. of Geometric Modeling and Processing 2000, Hong Kong, April 10-12, 2000, pp. 364-372.

6. G. Elber and M.-S. Kim, Geometric constraint solver using multivariate rational spline functions, in Proc. of the Sixth ACM Symposium on Solid Modeling and Applications 2000, Ann Arbor, Michigan, June 6-8, 2001, pp. 1-10.

7. H.-S. Heo, The Intersection of Ruled and Ringed Surfaces, Ph.D. Thesis, Dept. of Computer Science, POSTECH, February 2000.

8. H.-S. Heo, M.-S. Kim, and G. Elber, The intersection of two ruled surfaces, Comput. Aided Design 31, 1999, 33-50.

9. J. Hoschek and D. Lasser, Fundamentals of Computer Aided Geometric Design, A. K. Peters, Wellesley, MA, 1993.

10. J. Johnstone, A new intersection algorithm for cyclides and swept surfaces using circle decomposition, Comput. Aided Geom. Design 10, 1993, 1-24.

11. K.-J. Kim, M.-S. Kim, and K. Oh, Torus/sphere intersection based on a configuration space approach, Graphical Models Image Process. 60, 1998, 77-92.

12. M.-S. Kim, The intersection of simple sweep surfaces, in Proc. of the Riken Symposium on Geometric Processing for Innovative Applications, Tokyo, July 21, 2000, pp. 1-17.

13. M.-S. Kim and G. Elber, Problem reduction to parameter space, in The Mathematics of Surfaces (R. Cipolla and R. Martin, Eds.), Vol. 9, pp. 82-98, Springer-Verlag, London, 2000.

14. T. Maekawa, N. M. Patrikalakis, T. Sakkalis, and G. Yu, Analysis and applications of pipe surfaces, Comput. Aided Design 15, 1998, 437-458.

15. R. Martin, J. de Pont, and T. Sharrock, Cyclide surfaces in CAD, in The Mathematics of Surfaces I (J. Gregory, Ed.), pp. 253-267, Oxford University Press, Oxford, 1986.

16. J. Miller and R. Goldman, Geometric algorithms for detecting and calculating all conic sections in the intersection of any two natural quadric surfaces, Graphical Models Image Process. 57, 1995, 55-66.

17. T. Nishita and H. Johan, A scan line algorithm for rendering curved tubular objects, Proc. Pacific Graphics' 99, Seoul, Korea, October 5-7, 1999, pp. 92-101. 
18. N. Patrikalakis and T. Maekawa, Intersection problems, in Handbook of Computer Aided Geometric Design (G. Farin, J. Hoschek, and M.-S. Kim, Eds.), Elsevier, Amsterdam, 2002.

19. M. Peternell and H. Pottmann, Computing rational parametrizations of canal surfaces, J. Symbolic Comput. 23, 1997, 255-266.

20. J.-K. Seong, M.-S. Kim, and G. Elber, Ruled tracing of ringed surfaces, in preparation, 2001.

21. C.-K. Shene and J. Johnstone, On the lower degree intersections of two natural quadrics, ACM Trans. Graphics 13, 1994, 400-424.

22. J. van Wijk, Ray tracing objects defined by sweeping a sphere, Eurographics ' 84, pp. 73-82, North-Holland, Amsterdam, 1984. 